\title{
Balance disorders and hypothyroidism: A rare cause worth remembering
}

\author{
Ayhan Kull ${ }^{1}$, Arzu Bilen², Nuray Bilge ${ }^{3}$, Köksal Sarıhan ${ }^{1}$, Hülya Uzkeser ${ }^{1}$, Ramazan Dayanan², \\ Fatih Baygutalp ${ }^{1}$ \\ 1 Department of Physical Medicine and Rehabilitation, Medical Faculty of Atatürk University, Erzurum, Turkey \\ 2 Department of Endocrinology and Metabolic Diseases, Internal Medicine, Medical Faculty of Atatürk University, Erzurum, Turkey \\ 3 Department of Neurology, Medical Faculty of Atatürk University, Erzurum, Turkey \\ Ayhan Kul, ORCID: 0000-0003-1313-9469 \\ Arzu Bilen, ORCID: 0000-0001-9017-8344 \\ Nuray Bilge, ORCID: 0000-0002-9328-1678 \\ Köksal Sarıhan, ORCID: 0000-0002-5010-4591 \\ Hülya Uzkeser, ORCID: 0000-0002-1364-2657 \\ Ramazan Dayanan, ORCID: 0000-0003-0475-6203 \\ Fatih Baygutalp, ORCID: 0000-0002-7344-584X
}

\begin{abstract}
Objective: This study aimed to evaluate balance disorders and potential risk factors for falling in patients with hypothyroidism, as well as postural stability and the risk of falling.
\end{abstract}

Methods: The participants' sex, age, body mass index (BMI), falls history in the past one year, and Falls Efficacy Scale - International (FES-I), Neuropathic Pain Diagnostic questionnaire (DN4) and clinical symptoms were recorded. The stability index (SI), weight distribution index (WDI) and falls risk analysis were performed for postural stability by objective computerised dynamic posturography.

Results: 50 patients were matched for age and sex (mean age:41.7 \pm 11 years; age range:19-61 years) with 47

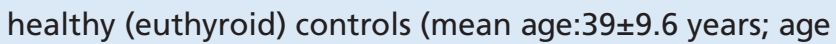
range:23-60 years). All SI, HL-WDI and falls risk scores, except FES-I, DN4 and NO-SI, were significantly higher in the patient group. In addition, other symptoms were present at a higher rate, with the exception of morning stiffness $(p<0.05)$. There was no significant association between sex, age, BMI, FES-I, DN4, serum FT4 and TSH levels, clinical symptoms and WDI values and the risk of falls $(r<0.3$ and $p>0.05)$. However, there was a slight positive correlation between the existence of paraesthetic symptoms, a history of falling, and the overall SI and HLWDI values $(r>0.3$ and $p<0.05)$.

Conclusions: It was determined that, as postural stability is affected in cases of hypothyroidism, balance becomes disordered and the risk of falls increases. This increase in the risk of falls was associated with being hypothyroid, but not with FT4 or TSH levels or the other factors evaluated.

Keywords: Hypothyroidism, postural balance, falling. 
Hypothyroidism is one of the most prevalent endocrine disorders and has a frequency in the general population of between 0.5 and $1 \%$. Hypothyroidism can affect multiple physiological systems, but especially affects the central nervous system, the peripheral nerves and muscles and may cause various symptoms and signs within the neuromuscular system. These signs range from various myopathies and entrapment mononeuropathy causing neuropathic pain in a glove-stocking distribution to a sensorimotor polyneuropathy, and can give a varying clinical picture consisting of symptoms like muscle and joint pain, cramp, weakness and fatigue..$^{[1]}$ Besides its strong association with the risk of falls, hypothyroidism is known to be a major cause of cerebellar and gait ataxias. ${ }^{[2,3]}$ It is known that the frequency of neuropathy is between 10 and $70 \%$, and that of myopathy between 20 and $80 \%$, in cases of hypothyroidism. ${ }^{[4]}$ Nonetheless, what is less recognised as a consequence of hypothyroidism, is the fact that, depending on the severity and duration of thyroid deficiency, various parts of the vestibular system, which play a key role in maintaining balance and postural stability, may be affected, resulting in vertigo, dizziness and other similar problems. ${ }^{\left[{ }^{[j]}\right.}$

Polyneuropathies and other neuromuscular symptoms found in patients with hypothyroidism include insufficiency of motor and sensory function secondary to profound loss of superficial, vibrational and proprioceptive sensation, and may cause disordering of static and dynamic postural stability and hence increase the risk of falls. ${ }^{[6,7]}$ Despite the fact that a great many studies have been undertaken assessing postural stability and balance in patients with diabetes mellitus, one of the key causes of neuropathy, as far as the authors are aware, this kind of study has yet to be undertaken in patients with hypothyroidism. ${ }^{[6]}$ Such a study, by clarifying postural stability, falls risk and associated risk factors in adults with hypothyroid illness would contribute to their recognition, treatment and management.

In our study, we aim to assess postural stability and the risk of falls in adult patients with known hypothyroidism by means of an objective computerised system.

\section{Materials and Methods}

This cross-sectional study was undertaken between November 2018 and April 2019 by the Physical Medicine and Rehabilitation (PMR), Neurology and Endocrinology divisions of the Atatürk University School of Medicine. The study protocol received ethical approval from the Medical Faculty of Atatürk University Ethics Committee
(29.11.2018/7-1). Informed consent was obtained in writing from each participant. The study was conducted in accordance with the principles of the Declaration of Helsinki. The patients included in the study had a diagnosis of either subclinical or overt hypothyroidism according to endocrinological assessment, and were not confirmed to have any previous pathology in terms of postural stability or being at risk of falls. They received detailed clinical investigation by neurology specialists and neurological examination. Electromyography (EMG) did not indicate the existence of a polyneuropathy. The exclusion criteria for the study were: being unable to tolerate posturographic assessment or being unable to co-operate with it; having a previous history of orthopaedic or spinal surgery; suffering from a muscle disorder, movement disability, motor insufficiency, rheumatological disorders, degenerative osteoarthritis or diabetes mellitus; auditory or visual disorders; and any other type of pathology that could otherwise account for having a polyneuropathy. The control group consisted of individuals with normal thyroid function, and with no disorder that might cause balance problems.

To evaluate thyroid functions, free T4 (FT4; normal range:0.61-1.12 $\mathrm{ng} / \mathrm{dl}$ ) and thyroid stimulating hormone (TSH; normal range:0.34-5.6 mIU/ml) levels were measured by the chemiluminescent enzyme immunoassay method. A level of TSH $>5.6 \mathrm{uIU} / \mathrm{ml}$ or FT $4<0.6 \mathrm{ng} / \mathrm{dl}$ was considered overt hypothyroidism, TSH $>5.6 \mathrm{uIU} / \mathrm{ml}$ with FT4 in the normal range was considered subclinical hypothyroidism.

The sex, age, body mass index (BMI), any history of falls in the last year, the score on the Falls Efficacy Scale - International version (FES-I), score on the neuropathic pain questionnaire (DN4) and clinical symptoms (weakness, tiredness, morning stiffness, exercise intolerance, muscle and joint pain, diffuse pain and paraesthesia etc.) was recorded for all participants in the study. None of the patients stated they were using any medication which may cause balance problems. In addition, 25 of the patients were receiving thyroid hormone replacement therapy at a dosage of between 50-150 $\mu \mathrm{g}$.

The Falls Efficacy Scale - International version (FES-I) is a questionnaire based on self-reporting that allows quantification of the level of concern about falls whilst undertaking daily activities. The reliability and validity of the Turkish version of FES-I has previously been evaluated by Ulus et al. ${ }^{[8]}$ The DN4 scale consists of ten questions, each worth one point. Seven questions concern symptoms, 
whilst three are scored on physical examination. A total score of 4 or above is considered indicative of neuropathic pain. The Turkish language adaptation of the scale has been assessed for validity and reliability by Çevik et al. ${ }^{[9]}$

The Tetrax Interactive Balance System (Sunlight Medical Ltd, Tel Aviv, Israel) was used to assess both postural stability and to perform the falls risk analysis, in both cases and controls. The technique used was that outlined in the user guide for the device. ${ }^{[10]}$ In the Tetrax system, posturographic evaluation consists of a detailed investigation that allows calculation of the balance and falls risk indices. There are two configurations. Changes made to the centre of gravity are assessed in the stability index (SI). Fourier transformations are applied to the four independent wave signals gathered from the device's four platforms to separate out 8 frequency bands. The weight distribution index (WDI) is calculated from the footplate data, and compares the way the weight is distributed between left and right feet and between the heels and toes. By using the four measurements described above, and comparing them in eight separate positions, the postural performance analysis can be carried out. These 8 different positions are as follows: eyes open and closed, with head held upright (NO, $\mathrm{NC}$ ); eyes open and closed, head down, whilst standing on the foot pads (PO, PC); head turning to right and left whilst the eyes are closed (HR, HL); and eyes closed whilst extending and flexing the neck to the full extent ( $\mathrm{HB}, \mathrm{HF})$. Using all these postural measurements, the various parameters were then calculated. The parameters output from the Tetrax device may be used to calculate a falls index, which reflects the patient's risk of having a fall. This index takes into account the various mechanisms which normally should prevent falling. The value of the index is between zero and 100, allowing risk stratification into low (score of 35 or below), moderate (score between 36 and 57) and high (between 58 and 100) risk. ${ }^{[11]}$

\section{Statistical Analysis}

The $G^{*}$ Power application was used to claculate the minimal sample size needed. This indicated that a minimum of 31 were needed as participants in the patient and control groups for the $95 \%$ confidence interval. A sample size of 50 for both the cases and controls was chosen in view of probable drop-outs from the study. Three volunteers from the control group left the study at the beginning because they were unwilling to provide blood samples. At the end of the study, 50 healthy volunteers and 47 cases completed the protocol.
The SPSS v. 23 statistical software package (SPSS Inc, Chicago IL, USA) was used for all statistical analyses. The Kolmogorov-Smirnov test was used to assess the normality of the data. The descriptive statistics generated were the mean \pm standard deviation for normally distrubuted data, the median (minimum-maximum) for non-normally distributed data and the number (as a percentage) for categorical data.

The independent samples $t$ test and the Mann-Whitney $\mathrm{U}$ test were employed in group comparisons. The Chisquare test was used for $2 \times 2$ comparisons of variables of categorical type.

\section{Results}

In the cases group, 50 individuals participated, with a mean age of $41.7 \pm 11$ years (range 19-61 years), of which 5 were males and 45 females. In the control group, matched for age and sex, 47 euthyroid individuals took part, with a mean age of 39 \pm 9.6 years (range 23-60 years), of which 11 were males and 36 females. See Table 1.

The demographic and clinical characteristics, postural stability parameters and values for the risk of falling for both the cases and healthy controls are given in Table 1 . There was no significant difference between cases and controls, either in terms of age or gender $(p>0.05)$, but the $\mathrm{BMI}$ of individuals in the cases group was higher at the level of statistical significance $(p<0.05)$. The history of falls in the last 1 year was similar in both groups, with six hypothyroid individuals having a history of falling 14 times within the previous one year $(\mathrm{p}>0.05)$. FES-I scores were higher in the patient group, and this was a statistically significant difference between the groups $(\mathrm{p}<0.05)$. Mean FT4 and TSH levels in the patient group were $0.8 \pm 0.2 \mathrm{ng} / \mathrm{dl}$ and $9.3 \pm 12.2 \mathrm{mIU} / \mathrm{ml}$, respectively. The control group's values for FT4 and TSH were $0.9 \pm 0.2 \mathrm{ng} / \mathrm{dl}$ and $1.8 \pm 1 \mathrm{mIU} / \mathrm{ml}$, respectively. The FT4 level in the cases group was significantly lower, and the TSH significantly higher $(\mathrm{p}<0.05)$. Coming to the evaluation of the groups in terms of neuropathic pain and clinical symptoms, it was seen that the values obtained on the $\mathrm{DN} 4$ scale were significantly higher $(\mathrm{p}<0.05)$, as were all other symptoms except morning stiffness in the group with hypothyroidism $(\mathrm{p}<0.05)$. The overall SI and WDI scores obtained for postural stability in the groups are shown for the eight positions. There was a significant difference between the groups $(\mathrm{p}<0.05)$ in the other SI values obtained, apart from NO-SI, but for the WDI values obtained, only HL-WDI was significantly dif- 


\begin{tabular}{|c|c|c|c|c|c|c|c|c|c|c|c|}
\hline & \multicolumn{5}{|c|}{ Patients $(n=50)$} & \multicolumn{4}{|c|}{ Controls $(n=47)$} & \multirow[b]{2}{*}{ Min-Max } & \multirow[b]{2}{*}{ p } \\
\hline & $\mathbf{n}$ & $\%$ & Mean \pm SD & Median & Min-Max & $\mathbf{n}$ & $\%$ & Mean \pm SD & Median & & \\
\hline Gender (M/F) & $5 / 45$ & & & & & $11 / 36$ & & & & & 0.077 \\
\hline Age (year) & & & $41.7 \pm 11$ & & & & & $39 \pm 9.6$ & & & 0.196 \\
\hline BMI $(\mathrm{kg} / \mathrm{m} 2$ & & & $29.1 \pm 5$ & & & & & $26.5 \pm 3.6$ & & & $0.04 *$ \\
\hline Falls history & & 12 & & & & & 4 & & & & 0.068 \\
\hline FES-I & & & $25.4 \pm 8.8$ & & & & & $20.3 \pm 6$ & & & $0.001 * *$ \\
\hline $\begin{array}{l}\text { Serum FT4 level } \\
\text { (ng/dl) }\end{array}$ & & & $0.8 \pm 0.2$ & & & & & $0.9 \pm 0.2$ & & & $0.04 *$ \\
\hline $\begin{array}{l}\text { Serum TSH level } \\
(\mathrm{mlU} / \mathrm{ml})\end{array}$ & & & $9.3 \pm 12.2$ & & & & & $1.8 \pm 1$ & & & $0.001 * *$ \\
\hline DN4 & & & $3,5 \pm 2,2$ & & & & & $1.1 \pm 1.2$ & & & $<0.001 * *$ \\
\hline \multicolumn{12}{|c|}{ Clinical Symptoms (\% frequency) } \\
\hline Weakness & & 82 & & & & & 45 & & & & $<0.001$ ** \\
\hline Fatigue & & 78 & & & & & 45 & & & & $0.001 * *$ \\
\hline Morning Stiffness & & 4 & & & & & 10 & & & & 0.211 \\
\hline Intolerance of exercise & & 46 & & & & & 0 & & & & $<0.001$ ** \\
\hline Muscle pain & & 78 & & & & & 2 & & & & $<0.001$ ** \\
\hline Arthralgia & & 72 & & & & & 30 & & & & $<0.001$ ** \\
\hline Widespread pain & & 60 & & & & & 25 & & & & $<0.001$ ** \\
\hline Paresthesia & & 68 & & & & & 13 & & & & $<0.001$ ** \\
\hline \multicolumn{12}{|c|}{ Postural stability parameters } \\
\hline \multicolumn{12}{|l|}{ SI values } \\
\hline NO-SI & & & & 15.9 & $9.3-29.5$ & & & & 14.9 & $10-78.7$ & 0.262 \\
\hline NC-SI & & & & 23.3 & $14.2-50.1$ & & & & 19.4 & $13.7-32.1$ & $<0.001 * *$ \\
\hline PO-SI & & & & 18.1 & $11.4-53.9$ & & & & 16.6 & $10-38.1$ & $0.022 *$ \\
\hline PC-SI & & & & 29.4 & $13.1-65.9$ & & & & 25.1 & $17.9-44.3$ & $<0.001$ ** \\
\hline HR-SI & & & & 23 & $12.6-49.6$ & & & & 19.3 & $11.5-42.5$ & $0.007 * *$ \\
\hline HL-SI & & & & 25.5 & $12.1-56.6$ & & & & 21.3 & $14.5-48.1$ & $<0.001 * *$ \\
\hline HB-SI & & & & 27.4 & $12.6-74.6$ & & & & 20.9 & $14.8-39.3$ & $<0.001$ ** \\
\hline HF-SI & & & & 28.1 & $16-56.8$ & & & & 19.9 & $13.6-36.1$ & $<0.001 * *$ \\
\hline \multicolumn{12}{|l|}{ WDI values } \\
\hline NO-WDI & & & & 6.2 & $1.7-16$ & & & & 5.3 & $1.1-12.6$ & 0.431 \\
\hline NC-WDI & & & & 6.4 & $1.2-15.5$ & & & & 4.9 & $1.9-11.5$ & 0.073 \\
\hline PO-WDI & & & & 8.2 & $1.4-17.5$ & & & & 7 & $2.1-21.4$ & 0.321 \\
\hline PC-WDI & & & & 6.8 & $0.8-14.2$ & & & & 6.6 & 1.4-17.1 & 0.718 \\
\hline HR-WDI & & & & 6 & $0.5-19$ & & & & 5.2 & $1.8-14.1$ & 0.083 \\
\hline HL-WDI & & & & 6.6 & $2.2-17.4$ & & & & 4.9 & $1.8-13.6$ & $0.01 * *$ \\
\hline HB-WDI & & & & 6.2 & $1.4-17.8$ & & & & 6.1 & $1.2-13.5$ & 0.127 \\
\hline HF-WDI & & & & 6 & $1.6-21.5$ & & & & 5.3 & $1.4-13$ & 0.330 \\
\hline $\begin{array}{l}\text { Fall risk assessment } \\
(0-100)\end{array}$ & & & & 53 & $0-100$ & & & & 30 & $4-92$ & $<0.001 * *$ \\
\hline \multicolumn{12}{|l|}{ Fall risk category } \\
\hline Low & $14 / 50$ & 28 & & & & $31 / 47$ & 66 & & & & \\
\hline Moderate & $16 / 50$ & 32 & & & & $11 / 47$ & 23 & & & & \\
\hline High & $20 / 50$ & 40 & & & & $5 / 47$ & 11 & & & & \\
\hline
\end{tabular}

SD: Standard deviation; Min: Minimum; Max: Maximum; M: Male, F: Female, BMI: Body mass index; FES-I: Falls Efficacy Scale International, SI: Stability Index, WDI: Weight Distribution Index, NO: open eyes- head neutral, NC: closed eyes- head neutral, PO: open eyes-sponge pad under the feet, PC: closed eyes-sponge pad under the feet, HR: closed eyes - head rotated to the right, HL: closed eyes - head rotated to the left, HB: closed eyes - head fully extended, HF: closed eyes - head fully flexed, *. p<0.05 was considered as statistically significant between patient and control groups, ${ }^{* *}$. $p<0.01$ was considered as statistically significant between patient and control groups. 
ferent $(\mathrm{p}<0.05)$. In the falls risk analysis performed by the computerised system, the fall risk category was found to be moderate (risk assessment: 53) in the cases group and was higher than the controls group at the level of statistical significance $(\mathrm{p}<0.05)($ Table 1$)$.

In the cases group, there were no significant correlations between sex, age, BMI, FES-I, DN4, FT4 or TSH level, clinical symptoms and the risk of falling $(r<0.3$; $\mathrm{p}<0.05$ ). For the risk of falling, there was a weak positive correlation only amongst symptoms of paraesthesia and previous history of falls ( $r>0.3$ with $\mathrm{p}<0.05$ and $\mathrm{r}=0.281$ with $\mathrm{p}<0.05$, respectively). However, for postural stability and all values of SI, there was a significant positive association $(r>0.03$ with $\mathrm{p}<0.05)$. There was no demonstrable association between the values obtained for WDI and the risk of falling, except in the case of HL-WDI ( $r<0.3$ with $p>0.05$ ). In addition, the risk of falling was discovered to be at a low level in the control group, and a significant positive correlation was found between the risk of falling and all SI values $(\mathrm{p}<0.05)$, (Table 2$)$.

\section{Discussion}

By using the objective computerised technique, postural stability was shown to be disordered and the risk of falling increased in individuals with hypothyroidism. This increase in the risk of falling had an association with hypothyroidism, but not with the levels of FT4 or TSH, or the other factors under investigation.

Balance and postural stability is a complex physiological function, encompassing various neuromuscular processes. Sensory input, sensory evaluation and the neuromuscular response need to be controlled. Sensory input involves the vestibular, visual and proprioceptive systems. For an effective motor response, an intact neuromuscular system and sufficient muscular strength are needed. ${ }^{[12]}$ Hypothyroidism causes slowing of metabolic pathways, reduction in ATP synthesis, the inability to break down glycosaminoglycans, malfunction of the Na-K pumps and thus damage to axonal transport. It therefore leads to the neuromuscular and neurological problems that can be observed in the disorder. Hence hypothyroidism may be a cause for impairment of postural stability and an increase in the risk of falls occurring. ${ }^{[13]}$

Factors that increase the risk of falling include advanced age ( $>65$ years), being female, past falls and a fear of falling, living alone, having known medical problems, physical condition, impaired cognitive function, insufficient physi- cal activity, and environment, amongst multiple others. ${ }^{[14]}$ For this study, the age and sex distribution of the groups were similar, the cases were aged 41 years on average, and there were no additional medical conditions which might impair balance or increase the risk of falling. Thus no correlation was discovered amongst them with the risk of falling. Although the mean BMI values of both groups were close and in both cases indicated the subjects were overweight, there was a significant difference between them. However, the authors do not consider this situation to be clinically significant since it is not amongst the risk factors associated with falling. Although the history of falls was higher in the patient group, the result lacked statistical significance. FES-I values were, however, found to be significantly higher. Despite this, a relationship with the risk of falling was not established. This situation may be attributed to clinical symptoms insufficient for neuropathy, the absence of a polyneuropathy according to EMG results, and the fact that participants were not of advanced age.

Although the pathogenesis of polyneuropathy in hypothyroidism has not yet been completely elucidated, it has been linked to the accumulation of mucopolysaccharides in the endo- and perineurium, segmental demyelination, aggregation of glycogen, axonal degeneration and similar mechanisms of injury. ${ }^{[15-16]}$ Neuropathy eventually results in superficial and deep sensory losses such as proprioceptive and vibration, and this sensory loss may cause the impairment of balance and postural stability, with a consequently raised risk of falling. As far as the authors are aware, there has been no study undertaken on postural stability and the risk of falling utilising an objective methodology in hypothyroid patients whose electrophysiological test results were normal and who lacked polyneuropathy. Diabetes mellitus is one of the leading causes of polyneuropathy and multiple studies concerning postural stability, balance problems and the risk of falls exist in the literature. In diabetes mellitus, disorders affecting the somatosensory system have been reported to produce postural problems, loss of balance and a subsequent increase in the risk of falls. ${ }^{[13,17-18]}$ In addition, electrophysiological studies indicate that diabetic patients, even if they do not have a polyneuropathy, may lose their balance. ${ }^{[19]}$ From this study, postural stability and the risk of falling can be seen to be affected in hypothyroid patients, even where polyneuropathy is not present.

Our research agrees with the literature in showing that, in hypothyroidism, the incidence of cramp rises ${ }^{[1,10-}$ 
Table 2. The correlation between demographic, clinical, stability index and weight distribution index values with fall risk.

\begin{tabular}{|c|c|c|c|c|}
\hline & \multicolumn{2}{|c|}{ B12 patients with deficiency (fall risk) } & \multicolumn{2}{|c|}{ Healthy controls (fall risk) } \\
\hline & $r$ & p & $r$ & p \\
\hline Age (year) & 0.206 & 0.152 & 0.037 & 0.807 \\
\hline BMI $(\mathrm{kg} / \mathrm{m} 2)$ & 0.198 & 0.168 & 0.321 & $0.028^{*}$ \\
\hline Falls history & 0.281 & $0.048^{*}$ & 0.248 & 0.93 \\
\hline FES-I & 0.170 & 0.238 & 0.243 & 0.099 \\
\hline Serum FT4 level (ng/dl) & -0.086 & 0.551 & -0.348 & 0.017 \\
\hline Serum TSH level (mIU/ml) & -0.099 & 0.495 & -0.156 & 0.295 \\
\hline DN4 & 0.155 & 0.281 & -0.214 & 0.149 \\
\hline \multicolumn{5}{|l|}{ SI values } \\
\hline NO-SI & 0.411 & $0.003 *$ & 0.378 & $0,009 *$ \\
\hline NC-SI & 0.566 & $<0.001 * *$ & 0.720 & $<0.001$ ** \\
\hline PO-SI & 0.403 & $0.004 *$ & 0.658 & $<0.001$ ** \\
\hline PC-SI & 0.656 & $<0.001 * *$ & 0.518 & $<0.001$ ** \\
\hline HR-SI & 0.720 & $<0.001 * *$ & 0.701 & $<0.001$ ** \\
\hline HL-SI & 0.761 & $<0.001 * *$ & 0.707 & $<0.001$ ** \\
\hline HB-SI & 0.800 & $<0.001 * *$ & 0.689 & $<0.001$ ** \\
\hline HF-SI & 0.792 & $<0.001 * *$ & 0.751 & $<0.001$ ** \\
\hline \multicolumn{5}{|l|}{ WDI values } \\
\hline NO-WDI & 0.139 & 0.334 & 0.174 & 0.242 \\
\hline NC-WDI & 0.157 & 0.275 & 0.197 & 0.185 \\
\hline PO-WDI & -0.073 & 0.616 & -0.054 & 0.717 \\
\hline PC-WDI & 0.080 & 0.579 & -0.065 & 0.663 \\
\hline HR-WDI & 0.146 & 0.313 & 0.205 & 0.168 \\
\hline HL-WDI & 0.291 & $0.041 *$ & 0.028 & 0.854 \\
\hline HB-WDI & 0.187 & 0.193 & 0.014 & 0.928 \\
\hline HF-WDI & -0.076 & 0.601 & 0.016 & 0.913 \\
\hline
\end{tabular}

SD: Standard deviation, BMI: Body mass index; FES-I: Falls Efficacy Scale International, SI: Stability Index, WDI: Weight Distribution Index, NO: open eyeshead neutral, NC: closed eyes- head neutral, PO: open eyes-sponge pad under the feet, PC: closed eyes-sponge pad under the feet, HR: closed eyes head rotated to the right, HL: closed eyes - head rotated to the left, HB: closed eyes - head fully extended, HF: closed eyes - head fully flexed, *. Correlation is significant at the 0.05 level, **. Correlation is significant at the 0.01 level.

${ }^{12]}$, complaints of fatigue are at a rate of $40-70 \%{ }^{[1,4,13]}$, and diffuse pain ${ }^{[4,11,14-15]}$ and paraesthesia increase in frequency. ${ }^{[6,15]}$ Even if, in theory, subclinical hypothyroidism is considered an asymptomatic disorder, our study agrees with the literature in revealing a clear increase in neuromuscular symptoms in such patients.

This study confirms the literature on hypothyroidism in showing a statistically significant rise in weakness, fatigue, exercise intolerance, muscle and joint aches, diffuse pain and symptoms of paraesthesia, in those with hypothyroidism, as compared to healthy controls. This was not shown for morning stiffness, however. However, with the exception of symptoms of paraesthesia, no correlation could be established between the risk of falling and these symptoms. There are studies available in the literature reporting that pain, weakness and fatigue have an adverse effect on balance [21-25]. The absence of a significant correlation in our study may arise from the fact that the cases group had no additional problems, were not advanced in age, possessed full muscular strength and may have exaggerated the symptoms to an extent, or for other similar reasons. It has been demonstrated in this study that complaints indicating paraesthesia, 
such as burning, numbness and tingling may increase the risk of falls through impairment of postural stability. ${ }^{[26]}$

In our study, there was a significant difference in all the values of SI apart from NO-SI, but only the HL-WDI scores were significantly different amongst the WDI values. In the study, SI values were higher in the cases group. This indicates in a numerical form the changes in the parameters affecting balance, i.e. stance, muscle control and compensation that cannot be seen clinically. Higher values of the SI indicate a higher degree of imbalance in an individual, whereas a low SI indicates better balance and stability. Furthermore, the positive correlation found between all the parameters measured in the SI and the risk of falling indicate that balance plays a key role in determining an individual's risk of falling. In particular, where the PO-SI is more impaired than the NO-SI, which is a more straightforward position and reference point, this may show us that the patients cannot utilise their somatosensory systems (touch, proprioception and vibration sense etc) and are affected by the illness. In terms of the second of our parameters, the WDI, when there is an asymmetric distribution between the right and left feet, we can observe interactions between the heels and toe regions and between the two sides. High WDI is indicative of pathology, whilst values approaching zero indicate maximal postural stability, and are often produced by mechanisms responsible for attaining compensatory equilibrium. Although there was no significant difference between the groups, higher WDI values in the patient group could indicate that the compensatory mechanisms (e.g. somatosensory, visual and vestibular) in patients work less well than in the control group. Potentially, had the patients had greater symptoms or comprised more individuals, the difference between them might have reached statistical significance. The raised HL-WDI value (which was statistically significant) found in the patient group may indicate an undetected orthopaedic problem affecting the feet. There was also a subset of individuals within the control group, who fell into the low risk category for falling. In this group, there was a positive correlation between values on the stability index and the risk of falling. This correlation has no clinical significance, however, since there is no need to take precautions in a group with such a low risk of falls.

The elevated risk of falling observed in this study in patients with hypothyroidism may, we believe, arise from defects in the visual, vestibular or proprioceptive systems causing an impulse disorder. Due to the moderate risk of falls shown to exist in patients with hypothyroidism, patients should do regular exercise and be cautious when walking or running. Furthermore, when they are in contact with or walking on slippery surfaces, they need to take the necessary precautions to avoid falling. Advancing age, as well as various vitamin deficiencies, a rise in symptoms of neuropathy, the increase in degenerative disorder and, specifically in women, osteoporosis, can lead to fractures and their complications linked to falls. Our study may contribute to awareness of these problems and how to prevent them and thereby raise the quality of life.

One of the limitations of the study may be accepted as arising from the fact that some of the hypothyroid patients were already taking thyroid replacement therapy, and the group was not divided into two groups, subclinical or overt hypothyroidism, which may have permitted a better analysis.

\section{Conclusion}

In this study, it was found that postural stability was impaired and the risk of falling increased in hypothyroid patients. This increase in the risk of falling correlated with hypothyroidism but not with the level of FT4 or TSH, nor the other factors investigated. Accordingly, this increase in risk of falls may have occurred as a result of neuronal degeneration due to disease, even though the existence of a polyneuropathy is not reflected in electrophysiological tests. In addition, hypothyroidism should be considered as a possible cause of loss of balance, postural instability and increased risk of falls in patients without evidence of vestibular pathology.

Ethics Committee Approval: The study protocol received ethical approval from the Medical Faculty of Atatürk University Ethics Committee (29.11.2018/7-1).

Informed Consent: All patients provided written informed consent.

Author Contributions: Designing the study - A.K., A.B., K.S., H.U.; Collecting the data - A.K., A.B., N.B., K.S., H.U., R.D.; Analyzing the data - A.K; Writing the manuscript - A.K., N.B., F.B.; Confirming the accuracy of the data and the analyses - A.K., K.S., F.B.

Conflict of Interest: The authors have no conflicts of interest to declare.

Financial Disclosure: The authors declared that this study has received no financial support. 


\section{References}

1. Penza P, Lombardi R, Camozzi F, Ciano C, Lauria G. Painful neuropathy in subclinical hypothyroidism: clinical and neuropathological recovery after hormone replacement therapy. Neurol Sci 2009;30:149-51.

2. Kotwal SK, Kotwal S, Gupta R, Singh JB, Mahajan A. Cerebellar ataxia as presenting feature of hypothyroidism. Arch Endocrinol Metab 2016;60:183-5.

3. Verghese J, Holtzer R, Lipton RB, Wang C. Quantitative gait markers and incident fall risk in older adults. J Gerontol A Biol Sci Med Sci 2009;64:896-901.

4. Duyff RF, Van den Bosch J, Laman DM, van Loon BJ, Linssen WH. Neuromuscular findings in thyroid dysfunction: a prospective clinical and electrodignostic study. J Neurol Neurosurg Psychiatry 2000;68:750-5.

5. Rybak LP. Metabolic disorders of the vestibular system. Otolaryngol Head Neck Surg 1995;112:128-32.

6. Simmons RW, Richardson C, Pozos R. Postural stability of diabetic patients with and without cutaneous sensory deficit in the foot. Diabetes Res Clin Pract 1997;36:153-60.

7. van Deursen RWM, Simoneau GG. Foot and ankle sensory neuropathy, proprioception, and postural stability. J Orthop Sports Phys Ther 1999;29:718-26.

8. Ulus Y, Durmus D, Akyol Y, Terzi Y, Bilgici A, Kuru O. Reliability and validity of the Turkish version of the Falls Efficacy Scale International (FES-I) in community-dwelling older persons. Arch Gerontol Geriatr 2012;54:429-33.

9. Unal-Cevik I, Sarioglu-Ay S, Evcik D. A comparison of the DN4 and LANSS questionnaires in the assessment of neuropathic pain: validity and reliability of the Turkish version of DN4. J Pain 2010;11:1129-35.

10. Alkan Melikoğlu M, Bağcier F. Fall Risk and Related Factors in Systemic Lupus Erythematosus. Arch Rheumatol 2017;32:216-20.

11. Özdemir O, Gökçe Kutsal Y. Fall risk assessment of elderly by using posturography. Turk J Geriatr 2009;12:177-80.

12. Jones G. Posture. In: Kandel ER, Schwartz JH, Jessel TM, editors. Principles of Neural Science. New York, NY: McGraw-Hill; 2000. p. 816-31.

13. Mustapa A, Justine M, Mohd Mustafah N, Jamil N, Manaf H. Postural Control and Gait Performance in the Diabetic Peripheral Neuropathy: A Systematic Review. Biomed Res Int 2016;2016:9305025.
14. Lord SR. Falls. In: Halter JB, Ouslander JG, Studenski S, et al, editors. Hazzard's Geriatric Medicine and Gerontology. 7th ed. New York, NY: McGraw Hill Education; 2017. p. 1318-33.

15. Ferreira AA, Nazário JC, Pereira MJ, Azevedo NL, Barradas PC. Effects of experimental hypothyroidism on myelin function sheath structural organization. J Neurocytol 2004;33:225-31.

16. Khedr EM, El Toony LF, Tarkhan MN, Abdella G. Peripheral and central nervous system alterations in hypothyroidism: electrophysiological findings. Neuropsychobiology 2000;41:88-94.

17. Treede RD, Jensen TS, Campbell JN, et al. Neuropathic pain: redefinition and a grading system for clinical and research purposes. Neurology 2008;70:1630-5.

18. John SA, Solomon J, Mohan D. A Critical Review on the Normal Postural Control. POTJ 2015;8:71-5.

19. Brown SJ, Handsaker JC, Bowling FL, Boulton AJ, Reeves ND. Diabetic peripheral neuropathy compromises balance during daily activities. Diabetes Care 2015;38:1116-22.

20. Akarsu EA, Acar H, Ozer F, et al. Electromyographic Findings in Overt Hypothyroidism and Subclinical Hypothyroidism. Turk J Neurol 2013;19:128-33.

21. Treede RD, Apkarian AV, Bromm B, Greenspan JD, Lenz FA. Cortical representation of pain: functional characterization of nociceptive areas near the lateral sulcus. Pain 2000;87:113-9.

22. Leveille SG, Jones RN, Kiely DK, et al. Chronic Musculoskeletal Pain and the Occurrence of Falls in an Older Population. JAMA 2009;302:2214-21.

23. Morrison S, Colberg SR, Parson HK, et al. Walking-Induced Fatigue Leads to Increased Falls Risk in Older Adults. J Am Med Dir Assoc 2016;17:402-9.

24. Helbostad JL, Sturnieks DL, Menant J, Delbaere K, Lord SR, Pijnappels $\mathrm{M}$. Consequences of lower extremity and trunk muscle fatigue on balance and functional tasks in older people: A systematic literature review. BMC Geriatrics 2010;10:56.

25. Masud T, Morris RO. Epidemiology of falls. Age Ageing 2001;30:3-7.

26. Morimoto A, Sonoda N, Ugi S, et al. Association between symptoms of bilateral numbness and/or paresthesia in the feet and postural instability in Japanese patients with diabetes. Diabetol Int 2016;7:69-76.

This is an open access article distributed under the terms of the Creative Commons Attribution-NonCommercial-NoDerivs 3.0 Unported (CC BY- NCND3.0) Licence (http://creativecommons.org/licenses/by-nc-nd/3.0/) which permits unrestricted noncommercial use, distribution, and reproduc- tion in any medium, provided the original work is properly cited.

Please cite this article as: Kul A, Bilen A, Bilge N, Sarıhan K, Uzkeser H, Dayanan R, Baygutalp F. Balance disorders and hypothyroidism: A rare cause worth remembering ENT Updates 2019;9(3): 172-179. 